\title{
Rhétorique et éthique de la citation
}

\author{
PIERRE-LOUIS VAILLANCOURT
}

Au moment même où s'affaiblissaient, dans la recherche littéraire, les notions d'influence, d'emprunt et de fortune, bousculées par des préoccupations plus strictement formelles et synchroniques du texte, un certain nombre d'études, plus liées à la sémiotique qu'à l'histoire littéraire, accusaient un intérêt nouveau pour le problème de la citation et s'inscrivaient dans la foulée des considérations de la nouvelle critique sur les rapports, dans le texte, entre l'invention et l'imitation, sur la nature de la copie (Barthes) et sur l'intertextualité (Kristeva). Dans les actes du colloque de sémiotique tenu à Varsovie en 1968 , une section entière est dévolue à la citation. Dans un de ces articles, S. Morawski distingue quatre fonctions de la citation dans un texte: érudition, autorité, amplification, ornement. ${ }^{1}$ Cette approche formaliste inspirera Per Aage Brandt et D. Maingueneau dans les pages qu'ils consacreront à cette question. ${ }^{2}$

Analysant certains de ces travaux dans la perspective de la réception critique, Bernard Beugnot les compare aux réflexions faites à diverses époques sur cette question et présente un tableau érudit de la poétique de la citation, depuis les Grecs, où le contexte diachronique était fort différent, jusqu'à nos jours. ${ }^{3}$ Notant que les analyses récentes ne vont pas tellement plus loin que les anciennes, B. Beaugnot souligne bien que semblent s'engouffrer dans les études de la citation les notions proclamées périmées de fortune et d'influence.

A peine trois ans après cet article, dans lequel était formulé le voeu d'une histoire plus détaillée de la citation, paraissait l'ouvrage d'Antoine Compagnon: La Seconde Main ou le travail de la citation, ${ }^{4}$ où se trouve réaffirmée l'importance de Montaigne sur le rôle de la citation. A son tour, A. Compagnon "revisite" la dimension historique du problème; il s'attarde à certains types d'oeuvres où elle trouva le mieux à s'accomplir, comme dans les emblèmes, et il aborde longuement l'exégèse biblique.

La Renaissance est perçue comme une période transitoire entre une poétique médiévale de la copie, pour reprendre les propos de Roland Barthes dans Critique et vérité, ${ }^{5}$ et une régulation du discours à l'âge classique qui 
entraînera une dépréciation de la citation. Au Moyen Age, le texte devait être reconduit dans son intégrité et n'était retouché qu'en vue de plus d'intelligibilité. Que cet esprit de fidélité, comme le remarque Barthes, ait abouti à un résultat très souvent opposé, peu nous importe ici. Les instruments pratiques de cette fidélité affichée se trouveront bouleversés, et en quelque sorte résolus, par l'invention de l'imprimerie, laquelle favorisa de nouvelles attitudes et une éthique différente à l'égard du texte ancien.

Montaigne occupe une place privilégiée dans la problématique de la citation et $\mathrm{A}$. Compagnon suggère de l'étudier à la lumière de la théorie du signe à cette époque, contrairement aux études de $\mathrm{H}$. Friedrich et de $\mathbf{M}$. Metschies. A. Compagnon perçoit également, dans l'utilisation que fait Montaigne de la citation, une contestation de l'auctoriatas. Sans entrer dans ces considérations, reconnaissons à Montaigne le mérite d'avoir inscrit, à l'instar d'Erasme et de Ramus, la citation dans la méthode du discours et d'en avoir exploré diverses facettes. Cette diversité d'emplois permet d'évoquer, autour du nom de Montaigne, quelques-unes des nombreuses interrogations de la critique à propos de la citation. Montaigne soulève par exemple la question d'éthique par la rareté des références à ses sources. Les auteurs et les époques jugeront différemment cette omission. La critique contemporaine y voit un moyen de mieux attirer l'attention sur le contenu de l'énoncé, au détriment des conditions de l'énonciation, ou même un instrument de lutte contre la propriété des idées et du discours. Aussi reprendelle le procédé, dont elle abuse parfois. Montaigne apparait comme le héros/héraut de l'opposition aux diverses formes d'appropriation. Ce dernier, qui attribuait son recours fréquent aux textes anciens à sa "condition singeresse et imitatrice," ${ }^{\prime 6}$ eut été sans doute le premier surpris de voir sa "paresse" investie d'une telle mission, à une époque où on ne connaissait pas le copyright et où on ne reconnaissait pas du tout la propriété des idées. Seul le plagiat, comme en témoignent les accusations de François Hotman contre Pierre Faber, était considéré comme indigne. ${ }^{7}$ Montaigne devait bien plutôt partager la satisfaction des auteurs de son temps lorsqu'ils se rencontraient aux jugements des Anciens et leur plaisir lorsqu'ils y trouvaient une confirmation de leurs idées.

Dans les Essais, présentés par leur auteur comme le résidu des “dépouilles de deux autres," se manifeste fréquemment un goût avoué de citer, d'imiter, d'emprunter, voire même de créer de toutes pièces de fausses citations. ${ }^{8} \mathrm{Ce}$ même goût d'étaler une érudition, sans nécessairement préciser les sources, se retrouve dans la littérature politique, en particulier dans les emblèmes et les blasons, comme le remarque A. Compagnon.

Cette complaisance à emprunter ne trouve pas de censeurs au début de la Renaissance, alors qu'à l'aube du siècle suivant, Alexandre-Paul Filière écrira tout un traité dont le titre constitue une profession de foi: Discours contre les citations du grec et du latin ès plaidoyers de ce temps ${ }^{9}$ et dont la 
parution marque à la fois l'apothéose d'une méthode et le début de sa décadence. L'affaiblissement de la pratique et de la connaissance de ces deux langues anciennes ne suffit pas à justifier ces changements dans les impératifs de la mode. Entre ces deux débuts de siècle, que nous posons comme balises historiques uniquement pour la commodité de l'argumentation, l'habitude d'emprunter et de citer a pâti en raison même de son succès.

Dans la littérature politique de la Renaissance se posait, avec autant d'acuité, sinon davantage en raison du contenu des oeuvres, le problème du respect des idées des auteurs anciens. Lorsque Juste Lipse fait paraître ses Politiques, où abondent les citations d'auteurs anciens, il commente sa méthode dans son épître $\mathrm{Au}$ Lecteur:

\begin{abstract}
Nous aussi mettons en avant, sous vostre support, ces Politiques, escrits d'une façon autre \& totalement nouvelle. Car nous les avons dressez d'un stile qu'on n'eust pas attendu: auquel je puis bien dire que tout est nostre, \& qu'il n'y a rien du nostre. Pource que l'Invention \& l'Ordre estant entierement de nous, toutesfois nous avons recueilli les mots des anciens auteurs, principalement des Historiens: c'est à dire, comme j'estime, des sources de la Prudence politique. L'ambition ni le vent de nouveauté ne nous a point poussez à cela, je le proteste franchement, mais l'affection de faire chose qui vous fust utile. Pouvois-je mieux rencontrer, que rassemblant tant de belles, vives \& salutaires sentences en un corps? Et quand je dirois le mesme, qui m'en croiroit, \& qui s'en seroit esmeu? Comme il importe beaucoup de quelle main part un trait ou un coup d'espée: autant en faut-il dire d'un propos notable pour lui donner entree au coeur, le poids de quelque authorité puissante $\&$ approuvee y sert beaucoup. Ceste authorité se trouve es anciens. Mais nous n'avons pas presenté des sentences nues ou esparses; de peur qu'elles ne s'espandissent, \& que ce ne fust (comme on dit) du sable sans chaulx: mais nous les avons dextrement cousues les unes aux autres, ou quelquesfois les avons jointes ensemble par le ciment de nos paroles. Somme, ainsi que les brodeurs font une piece de tapisserie avec du fil de diverses couleurs: ainsi nous avons basti de quelques milliers de parcelles un corps qui paroit comme tout d'une piece. D'avantage, j'ay prins la hardiesse de l'orner de figures \& divers traits de langage, pour adjouster de l'esprit \& de la vie à la couleur. ${ }^{10}$
\end{abstract}

Lipse reconnaît donc clairement à la citation la fonction d'autorité mais non celle d'ornement, puisqu'il s'attribue tout le mérite de l'ordre, de l'invention, de la disposition.

Les Politiques paraissent à Lyon en 1589, en latin, La seconde édition, en français, de Genève en 1613, contient une addition à l'épître $A u$ Lecteur intitulée Quelques avertissemens, ou precautions, dans laquelle Lipse répond à ses détracteurs et pose notamment le problème de la fidélité aux sources:

La seconde accusation semble devoir estre, ou avoir esté desja, contra ma fidelité. Vous n'alleguez pas bien, disent-ils, les sentences des auteurs, ni selon leur intention. Je n'ai peu me contenir de rire, oyant telle censure: car, pour dire le vrai, ces beaux controlleurs blasment ce que tout homme de bon esprit 
m'attribuera à louange. Pouvois-je ou devois-je faire autrement en ce recueil? Est-ce point un assemblage de diverses pieces, dans lequel tels destours sont libres \& louables à cause de ce qu'ils contienent? Qu'ils regardent les poetes, qui jadis \& de nostre temps se sont esgayez à faire des Centons. Seulement il convient donner ordre de ne faire servir les paroles des auteurs á maintenir quelque meschante ou fausse opinion. Mes censeurs ne me chargent pas de cela, ni ne le peuvent faire aussi. Au reste toute ceste accusation est legere \& vaine: car nous n'avons rien mis en avant qui fust pour aprester à rire (estant question de manier un argument grave \& serieux) que fort rarement: \& tout expres avons forclos toutes gaillardes pointes \& rencontres, si ce n'a esté d'avanture en quelques liaisons \& pour parfaire quelque sentence de grand poids. Car alors, n'ayans rien en main qui eust esté dit à propos par nos auteurs: il m'a esté loisible de tourner doucement \& proprement quelque mot. C'est chose permise en tel cas. Je ne veux pas nier, que quelquesfois je n'aye allegué les auteurs un peu autrement qu'ils n'ont escrit, ayant quelque peu changé les Cas $\&$ les Temps des Noms \& Verbes, pour rendre mon discours plus uni, qui sans cest expedient n'avoit point de grace. ${ }^{11}$

Cette justification embarrassée et tortueuse constitue une précieuse référence pour l'évaluation de la conscience littéraire de cette époque. L'existence d'une double tendance (ceux qui blâment ce que "tout homme de bon esprit" loue) à l'égard des emprunts, au début du XVII ${ }^{\mathrm{e}}$ siècle, se trouve confirmée. Le souci de la fidélité se manifeste par la mention exacte des sources, approuvée et pratiquée par Lipse lui-même, mais surtout par le maintien du sens initial des citations, quand elles sont mises au service d'idées nouvelles, principe qui fournit la base de leur argumentation aux détracteurs de Lipse. A côté de ce souci semble subsister une pratique moins sévère, plus laxiste, illustrée encore par Lipse. Celui-ci ne trouve pour la justifier que le recours à une éthique plus générale, comme de ne jamais faire servir une citation à une démonstration erronée, ou l'évocation des difficultés d'ordre stylistique que pose l'insertion des citations, à cause de l'écart temporel des textes. Le ton défensif adopté par Lipse semble cependant indiquer le renforcement des contraintes et des règles dans l'emploi des citations, confirmé par la parution à la même époque du traité de Filière.

Montaigne s'était déclaré satisfait d'avoir puisé principalement chez deux auteurs. Lipse n'hésite plus à recourir à toute la littérature antique et tente simplement de mettre un peu d'ordre et de suite à tous ces textes morcelés. Ce travail, en littérature politique, présente des analogies avec la constitution des florilègs, signalés par B. Beugnot, où se trouvaient groupées, le plus souvent par ordre alphabétique, les notions abordées par les Anciens. Cette forme de fragmentation sévit très tôt dans la littérature politique, où compte beaucoup la fonction d'autorité, mais elle reste en général soumise à une visée démonstrative. Ainsi pour appuyer une seule idée, Jean Bodin invoquera plusieurs auteurs: 
Brief, si nous cherchons l'authoritez, nous trouverons que les plus grands personnages qui furent onques ont tenu que la Monarchie est la meilleure: à sçavoir Homere, Herodote, Platon, Aristote, Xenophon, Plutarque, Philon, Apollonius, sainct Hierosme, Cyprien, Maximum Tyrius, plusieures autres. ${ }^{12}$

Et Bodin se livre souvent, à l'instar des écrivains politiques du temps, à une véritable mobilisation de citations. Les chapitres de l'ouvrage de Jean de Marnix, Résolutions politiques et Maximes d'Estat, au XVII ${ }^{\mathrm{e}}$ siècle, ${ }^{13}$ sont un véritable montage de citations diverses. Tite-Live, Tacite, Salluste, Aristote, Cicéron, Lucain, Plutarque, sont tour à tour invoqués dans un chapitre sur les séditions. Un développement peut être bâti de pièces éparses provenant de philosophes, d'orateurs, d'historiens et de juristes de tendance opposée.

C'est sous le patronage de tous les Anciens que Juste Lipse place son oeuvre:

Sages Payens, d'où prend son origine

Ce haut sçavoir, sous qui gist abatu

L'erreur qui a le monde combatu, . . . ${ }^{14}$

Pour souligner l'inconstance des hautes fortunes, Lipse utilisera tout à tour Tacite, Salluste, Polybe et Sénèque. A la limite, l'auteur antique ne sert plus qu'à valoriser l'emploi d'un adjectif ou d'un qualificatif. Lipse accuse le peuple d'être instable et sans jugement, selon Cicéron; de s'émouvoir et de croire tout facilement, selon Tacite; de suivre ses chefs comme des moutons, selon Sénèque; d'être soupçonneux et léger, encore selon Tacite. Même Grotius opérera de tels entassements. Recensant les autorités favorables au mensonge, il nommera Socrate, Platon, Xénophon, Cicéron "en quelques endroits," Plutarque, Quintilien et tous les Stoïciens. ${ }^{15}$

L'abus du procédé modifie les conditions de fidélité au texte ancien. Une idée qui devait une partie de son sens à un contexte original, se trouve modifiée lorsqu'elle se trouve assemblée à un nouvel univers conceptuel. Même s'il ne pense que fortifier et renchérir son propos par l'adjonction d'une citation, l'auteur de la Renaissance crée un nouveau sens simplement en la découpant et en l'extrayant de sa combinaison première. A la longue, le système n'a plus que l'apparence de fidélité aux Anciens. Et dans le déchirement évoqué par B. Beugnot entre l'appartenance originelle et les pressions du nouvel univers sémantique, la citation qui se trouve amalgamée à une série d'autres se trouve de plus en plus éloignée de son sens originel et elle contribue, même malgré elle, à l'instauration d'une pensée neuve.

Ainsi posé, le problème éthique de la citation, que nous définissons comme le plus grand respect possible de sons sens originel, semble se ramener à des considérations numériques, la fréquence d'emploi augmen- 
tant proportionnellement les chances d'infidélité. L'augmentation du nombre de citations, de Montaigne à Lipse, par exemple, contribuerait à la dévalorisation du procédé. Une telle explication ne saurait rendre compte de toute l'évolution du phénomène, car elle méconnaît notamment en quoi Montaigne avait déjà transformé le système par rapport aux usages du Moyen-Age et pourquoi un procédé, qui devient en défaveur, reste utilisé par un grand nombre d'écrivains.

Il importe donc de tenir compte de la situation propre à chaque auteur. Mais le degré d'occurrence des citations ne suffit pas à distinguer un auteur d'un autre. La marque de différenciation la plus singulière réside dans les modes d'insertion de la citation, c'est-à-dire dans les phénomènes d'énonciation provoqués par l'arrivée d'un "corps étranger" 16 qu'est la citation. L'étude de ces aspects, en apparence plus formaliste, n'élimine pas cependant les considérations d'éthique, à la condition d'entendre ce terme de façon restrictive, comme le plus grand respect possible du sens originel. Cette acceptation a pour but d'éliminer les jugements positifs ou négatifs et de refuser l'examen du texte ou de l'auteur d'une façon moraliste. Ainsi, s'il ne s'agissait que de la reconduction la plus stricte du texte originel, pourrions-nous considérer le plagiat comme le procédé éthiquement le plus pur, mais d'autres considérations nécessaires sur l'abolition des marques citatrices habituelles (ponctuation et guillemets) nous obligeraient à nuancer ce jugement. En somme, le mot éthique ne renvoie plus, comme dans la perspective traditionnelle, à la mention exacte de la source (oeuvre et auteur) mais à la capacité plus ou moins grande de maintenir le sens dans le voyage du texte, un peu comme des vêtements qu'on sort des valises et qui portent diversement, souvent selon leur mode de fabrication, les marques du déplacement subi.

A la genèse de l'emprunt se trouve l'intelligence du texte de référence. La juste compréhension du texte n'est pas une condition particulière de la citation. Elle existe tout autant dans l'emprunt des idées que des passages. Au cours des recherches faites pour une thèse portant sur l'influence de Sénèque dans la pensée politique de la Renaissance, de nombreuses différences apparurent entre les auteurs quant à leur évaluation de la pensée sénéquienne. Entre un respect très poussé de sens et son renversement se placent divers paliers, selon les glissements ou les amplifications de sens, lorsque par exemple la notion de bienfaisance est plutôt entendue comme celle de libéralité, ou la notion d'indifférence envers le peuple comprise comme celle de dédain. Ces extensions de sens proviennent autant de la présence d'un contexte nouveau que d'une manipulation du texte ancien. Elles constituent des transformations autant de l'esprit que de la lettre du texte. Les développements philosophiques ou les anecdotes présentent donc les mêmes difficultés de réajustement dans un texte nouveau que les citations, et les 
déplacements de sens y sont également liés bien souvent à la présence d'une nouvelle configuration textuelle.

La présence d'une nouvelle configuration textuelle n'est pas la seule cause d'un flottement possible dans le sens. L'existence d'un écart considérable entre les données politiques et sociales de la Renaissance et de l'Antiquité contribue à accroître la marge d'erreur. Certes, les écrivains politiques de la Renaissance citent aussi leurs contemporains, en particulier Machiavel pour le contredire, mais leur banque essentielle de textes reste antique. Or la fin du $\mathrm{XVI}^{e}$ siècle voit l'émergence de théories politiques fort distinctes défendues par des groupes aux intérêts opposés, comme les monarchomaques, les Politiques et les scolastiques. Toute notion antique est donc soumise à un tiraillement, par l'écart temporel et idéologique. L'idée d'absolutisme princier, qui doit être comprise dans le contexte de la Rome impériale pour Sénèque, se trouve appliquée à une réalité nouvelle et de plus traitée diversement selon les allégeances des auteurs de la Renaissance. Ceux-ci font une moisson aussi variée que ces trois savants dépeints par Sénèque, qui consultant la République de Platon, utilisent parfois un même thème, une même citation, une même sentence, à des fins démonstratives différentes. "Il ne faut pas s'étonner, écrit Sénèque, si chacun exploite le même sujet conformément à ses tendances. Dans le même pré, le boeuf cherche de l'herbe, le chien un lièvre, la cigogne des lézards." ${ }^{17}$ Une influence ne s'exerce jamais de façon univoque.

Ces différences de siècles et d'idéologies facilitent l'utilisation du critère de respect ou de non-respect du sens initial, plus aisément identifiable dans des textes à contenu polémique. Ce critère n'empêche pas cependant l'examen simultané des marques créées dans l'énonciation par la manipulation des citations. Les modalités d'inscription de ce "corps étranger" qu'est la citation jouent un rôle important dans l'appréciation de l'authenticité et de l'intégrité du sens initial. C'est le rapport du texte à ses deux contextes, l'ancien et le nouveau, qui crée la mesure de sa pertinence. Les formes les plus visibles de ce rapport sont celles de la redondance, avec l'un ou l'autre contexte, ou de la contradiction, avec les deux ou avec l'un seulement. Une citation peut donc être en situation de redondance à l'égard de son nouveau contexte mais de contradiction à l'égard de son ancien, ce qui constitue le moyen le plus sûr pour mesurer son infidélité. La citation réalise donc possiblement deux figures fondamentales de la rhétorique, la redondance et l'antithèse.

La linguistique contemporaine, suivie en cela par la psychanalyse et la critique littéraire, nous incite cependant à considérer plutôt la métaphore et la métonymie comme les modes fondamentaux de la création du sens et comme les mécanismes linguistiques nécessaires à la communication, par la substitution et par la combinaison d'un signifiant à un autre. La faculté de 
sélection, donc de substitution, et celle de combinaison, c'est-à-dire de mise en contexte, sont les deux modes essentiels d'arrangement des mots pour l'échange du sens, lequel constitue selon Jakobson un double procès, métaphorique et métonymique. ${ }^{18}$ Ces deux modes peuvent être également considérés comme les voies primordiales de l'insertion de la citation. Lorsque la citation, en effet, ne sert qu'à la redite ou à la répétition, lorsqu'elle est redondante par rapport au contexte dont elle reproduit les idées d'une manière plus pompeuse, plus connue grâce à la référence antique et par là plus convaincante, elle a en quelque sorte valeur de métaphore et elle joue le même rôle que cette figure majeure de la rhétorique. A côté de ce rapport fondé sur l'analogie apparaît une seconde forme, où la citation développe une notion accessoire plus ou moins importante en dépendance de la principale. Ce déplacement produit une sorte de métonymie où, comme dans la figure du même nom, une idée déjà affectée d'un sens et d'un contexte propre, est attribuée par extension à un contexte neuf. En général, seul le lecteur attentif distingue le second procédé du premier car la grande majorité des citations apparaissent comme des redondances de l'idée initiale. L'examen critique, en rappelant l'usage originel d'une notion, défait cette contiguiité apparente et dévoile le déguisement, l'écart métonymique.

Certes la redondance n'est jamais pure car le contexte dans le livre et dans l'histoire, n'est pas vraiment identique. Le simple découpage de même que la traduction créent déjà des différences. Néanmoins, la conformité des principes est parfois assez poussée pour que la citation soit davantage l'embellissement que le prolongement d'une idée. Dans une situation de convenance, la citation peut être introduite comme une simple comparaison, à l'aide des conjonctions habituelles. Si elle n'est pas traduite, la citation forme un appendice, une opposition. Elle y figure comme une incidence du texte français, c'est-à-dire une partie combinée mais non intégrante, n'affectant pas assez l'assertion première pour en modifier le sens. C'est ce procédé que Marnix utilise le plus souvent. Par exemple, après avoir écrit: "Un prince expérimente alors souvent qu'une injure soufferte \& patiente est occasion d'un plus grand bien" . . . il ajoute: "Saepe maiori fortunae locum fecit injuria." ${ }^{19}$ Le degré de variation entre les deux propositions est très faible, la seconde formant comme l'étendard de la première. Cette mise en relief est une tentation constante à la Renaissance et dans cet art se distinguent les esprits les plus cultivés, tels Lipse et Grotius qui excellent à retrouver des formules-clés analogues à leurs propos. C'est en suivant la recommandation de Sénèque: "Comme l'oeil du soleil couchant / Plus doucement nous va touchant," que Lipse s'excuse, dans son épître d'introduction des Politiques, des faiblesses de son oeuvre bâtie pendant une "longue langueur" au déclin de sa vie. ${ }^{20}$ Ce goût de la période versifiée persistera dans tout l'ouvrage de cet auteur capable de construire un traité par le brassage de matériaux antiques. Cette insertion élégante de vers en 
traduction produit d'ailleurs un effet plus raffiné que sa reproduction simple, comme dans cet exemple de Juan Marquez:

A raison de quoy Senecque disoit que le premier precepte gisoit à faire teste à

l'emulation. Ars prima regni est posse invidia pati (Medea). ${ }^{21}$

Si le rôle du vers est d'orner le discours, celui de l'anecdote sera de l'illustrer, c'est-à-dire de présenter non plus un discours analogue mais une situation convenant au raisonnement. L'exemple possédera les mêmes qualités que la sentence: la justesse, la brièveté, la force. A ce titre, l'anecdote rejoint le procédé métaphorique car elle constitue une parure du texte. Lorsque Marnix demande qu'on accorde les commissions aux messagers, il rappelle le passage de l'Apocoloquintose de Sénèque où Jupiter confie à Hercule, qui venait d'explorer la terre entière, de rechercher les antécédents de l'empereur Claude..$^{22}$ Marnix respecte à la fois l'anecdote et la digression morale, souvent soudées chez Sénèque, lorsqu'après avoir blâmé les princes de se conduire comme Alexandre et de faire les guerres sans nécessité, il rappelle le jugement négatif de l'auteur latin à l'égard des conquérants:

Qui remuaient non le monde mais leurs passions et ne s'arrêtaient plus tel le vent emporté et subissaient à la fin l'effet de leur violence. On ne retrouve pas son bonheur dans le malheur d'autrui. ${ }^{23}$

L'épisode sert de lien à deux commentaires qui sont en étroite correspondance. Chez Nicolas Faret, l'adéquation à certaines grandes argumentations de Sénèque sur la clémence et les bienfaits est si poussée que le texte, dépourvu du toute originalité, devient une copie. Se jugeant sans doute incapable de dépasser la disposition ou le style de son modèle, il en reproduit simplement les paroles, mais sans indiquer sa source. ${ }^{24} \mathrm{Ce}$ déguisement du plagiat doit lui paraître une faute moins grave et méprisable que la lourdeur d'une redite.

Si la redondance métaphorique, qui constitue le moyen le plus simple d'articulation du texte ancien, est un procédé relativement facile à reconnaître, il n'en pas de même de la métonymie, où le degré d'irrespect à l'égard du texte cité n'atteint pas toujours le contresens ou l'antithèse. Seule apparaît en position rhétorique d'antithèse la citation qui illustre une idée dérobée à un contexte et servant à l'expression d'une thèse opposée. Mais entre la redondance et l'antithèse subsiste tout en éventail d'interprétations et d'utilisations considérablement variables. Aussi antithèse et redondance s'opposent comme des cas limites, mais si la redondance peut être considérée comme l'unique réalisation de la métaphore, l'antithèse n'apparaît que comme l'une des modalités possibles de la métonymie. 'ces modalités intermédiaires peuvent à leur tour être décrites et désignées par les noms des figures de la rhétorique classique. 
A la frontière extrême du champ de la métonymie se trouve donc l'antithèse, qui suppose un renversement du sens initial. Un exemple d'un tel traitement est fourni par les emplois divers, par les écrivains de la Renaissance, du vers fameux prononcé par la nourrice de Médée dans la pièce de Sénèque: "Les haines qui se déclarent perdent le moyen de se venger." Ce conseil "machiavélique," bien digne des nourrices du théâtre de Sénèque, ne correspond pas évidemment aux thèses généreuses de l'auteur, développées dans les Bienfaits, sur la vengeance du prince. C'est en blâmant sa portée, conformément à l'esprit du message sénéquien, que Sir Robert Dallington présente ce vers dans un Aphorizme où il critique la volonté de faire le mal. ${ }^{25}$ Marnix cite le même vers, mais il en retient le sens propre et dissimule le contexte de l'énoncé dans un passage où il suggère au prince de ne montrer aucun ressentiment immédiat lors d'une offense et d'attendre, pour en obtenir réparation, d'avoir les moyens et les forces nécessaires. Exposant une situation où cette dissimulation peut être motivée, c'est-à-dire lorsque le prince découvre une conjuration, il écrit:

Il est bon d'appercevoir \& recognoistre les mauvaises intentions. Solem seaepe insidiarum remedium est, si non intelliganter. Tacite. Premierement afin de les pouvoir plus aisement prevenir \& dissiper, car dès qu'on manifeste son resentiment, on perd l'occasion d'en tirer raison. Professa odia perdunt vindictae locum. Seneque. ${ }^{26}$

Il amalgame ainsi le "tacitisme noir", ${ }^{27}$ au moralisme sénéquien. Dans les deux cas relevés, la citation produit une antithèse envers son contexte, soit antérieur, soit immédiat.

Les dialogues de théàtre dans Sénèque se prêtent facilement à ces dérivations, car les affrontements des protagonistes ne sont pas toujours conformes à l'éthique stoïcienne. Lipse ne résiste pas non plus à cette tentation d'ajuster un passage à une démonstration. Il transpose indûment les vers de Clytemnestre dans l'Agamemnon de Sénèque: "Quand les afaires sont fascheuses / Prenez les voyes plus scabreuses,'” pour justifier certaines guerres. Semblable sort est réservé à ces maximes fameuses, dont Sénèque n'a pas, il est vrai, l'exclusivité: "Qu'ils me haïssent pourvu qu'ils me craignent," "Qui ne sait dissimuler, ne sait régner,' et à cette autre invoquée par Thyeste pour refuser le pouvoir: "Fides, sanctitas, pietas privata bona sunt," dont Marnix et Dallington font un emploi cynique. ${ }^{28}$ Ces abus permirent de faire dire à un disciple du Portique comme Sénèque qu'un roi doit se méfier, se venger, dissimuler ses intentions et conserver le pouvoir à tout prix.

Toute une thématique a pu être manipulée de cette façon par la négligence du contexte. Dans son traité De la tranquillité de l'âme, Sénèque polémise contre la thèse d'Anthénodore favorable à la retraite. De larges extraits des 
arguments d'Anthénodore sont utilisés comme reflétant la pensée de Sénèque. Si bien que grâce à divers textes sénéquiens, Marnix et Lipse pourront se prononcer en faveur de la participation et Fitzherbert ${ }^{29}$ contre elle. La trahison est mince cependant, car Sénèque corrige et nuance sa doctrine dans son essai De l'oisiveté. Si des commentaires antagonistes peuvent surgir d'une source commune, c'est que la pensée de Sénèque est chatoyante et parfois contradictoire. De ces flottements naissent des interprétations divergentes: Philippe de Duplessis Mornay et François Hotman ${ }^{30}$ s'opposant à Grotius et à Marnix sur la question de la souveraineté, Althusius et Bodin différant de Grotius et Etienne de La Boétie ${ }^{31}$ sur la résistance au tyran, Fitzherbert contrariant Lipse et Marquez sur la problème de la Providence. Ces variations se trouvent facilitées par les bouleversements du système politique, économique et social à la fin du XVI ${ }^{e}$ siècle et au début du XVII ${ }^{\mathrm{e}}$ siècle. L'énorme écart temporel qui sépare Sénèque de ceux qu'il influence, étend aux notions, et non plus aux mots seulement, les changes d'impropriété, d'étrangeté. Les tentatives pour intégrer les citations ne dissimulent pas toujours leur air emprunté, l'accentuant même quand leur hétérogénéité n'est pas respectée.

L'antithèse n'est pas le seul procédé du champ de la métonymie. Quelquefois l'addition de la citation éveille dans l'esprit de l'auteur une idée accessoire et la citation sert de tremplin à l'élargissement de la notion. La citation peut aussi servir de notion complémentaire. Il s'agit bien là du sensus accomodatitius distingué par Dumarsais dans son chapitre: "Du Sens adapté ou que l'on donne par allusion":

Quelquefois on se sert des paroles de l'Ecriture Sainte ou de quelque auteur profane pour en faire une application particulière qui convient au sujet dont on veut parler, mais qui n'est pas le sens naturel et littéral de l'auteur dont on les emprunte, c'est ce qu'on appelle sensus accomodatitius, sens adapté. ${ }^{32}$

Les deux formes de "sens adapté" distinguées par Dumarsais sont la parodie - "on détourne, dans un sens railleur, des vers qu'un autre a fait dans une vue différente" - et le centon - "ouvrage composé de plusieurs vers ou de plusieurs passages empruntés d'un ou de plusieurs auteurs." 33

Plutôt que cette classification, de type trop exclusivement littéraire, nous retiendrons, pour désigner les citations dans lesquelles s'opère une transformation continue du sens, les figures de la métalepse et de catachrèse. La métalepse est dans la rhétorique une des formes particulières de la métonymie, avec l'antonomase et la synecdoque. Elle consiste à faire entendre une chose en exprimant ce qui l'amène ou ce qui la suit.

La citation apparaît donc en fonction de métalepse ${ }^{34}$ lorsqu'elle engendre une idée accessoire ou lorsqu'elle en forme une elle-même, ce qui est le cas le plus fréquent. La citation surgit alors à la suite d'un développement comme 


\section{4 / Renaissance and Reformation}

la réminiscence par un auteur d'une idée apparentée ou analogue. Parfois, la citation établit un pont entre deux notions dont la dissimilitude pourrait surprendre. Expliquant que les alliés ont le droit de se payer par du butin "parce que naturellement l'allié est tenu envers son allié à la réparation des dommages qui surviennent à cause de l'affaire commune ou publique," Grotius écrit encore:

Ajoutez ceci qu'on ne donne guère sa peine pour rien. Ainsi, dit Sénèque, on paye aux médecins le prix du service qu'ils nous rendent, le temps qu'ils nous consacrent en se détournant de leurs propres affaires. (Lib. VI, De Benef, c. 25.$)^{35}$

Les citations en fonction de métalepse sont parfois raccordées brutalement en finale de développement, et inscrites, même mal soudées, comme leur confirmation, donc sous les déguisements de la métaphore. Cet usage d'affecter une proposition particulière à une autre qui lui est relativement correspondante est très fréquent dans la littérature politique de la Renaissance. On transpose dans un texte qui déplore la rareté des bons conseillers les commentaires de Sénèque sur la rareté des Caton; les considérations sur la bienveillance du sage deviennent matière à digressions sur la libéralité du prince; le mépris du peuple sert à l'éloge de l'absolutisme. Si les effets de la colère sont qualifiés de désastreux en période de guerre, ils seront jugés également néfastes dans la répression des hérétiques. Le devoir de la sépulture sera associée aux impératifs généraux de l'humanité. La clémence vantée chez Auguste sera attribuée au peuple romain tout entier. La défense de la propriété privée sera transmuée en défense de la propriété publique.

Mesurer l'écart qui sépare une extension acceptable du sens d'un texte et un usage plus abusif, constitue une opération interprétative délicate, voire arbitraire, mais elle seule nous permet de distinguer la métalepse, désignation du premier procécé, de la catachrèse. La figure de l'hypallage aurait également pu être retenue pour la représentation de cette forme d'utilisation de la citation, car l'hypallage renvoie également à un changement du sens originel, à une dérivation ou même à un renversement, selon Fontanier. Néanmoins le mot catachrèse suggère davantage, par son origine étymologique, une forme abusive. ${ }^{36} \mathrm{La}$ catachrèse désigne tout usage citationnel où il y a abus de la signification primitive, lorsque l'auteur déduit de la citation un sens qu'elle ne souffre pas originellement, sans toutefois renverser ce sens comme dans l'antithèse. Plusieurs auteurs peuvent ainsi projeter un éclairage différent sur une même sentence. Thomas Fiztherbert emprunte un passage où Sénèque disait que d'un corps difforme pouvait naître un grand homme et l'ajoute à ses anathèmes contre l'avortement et l'infanticide. ${ }^{37}$ Plus tard, Marnix lie ce même passage à deux raisonnements distincts au sein de son ouvrage: d'abord dans une "Résolution" incitant le 
Prince à se méfier, pour le choix d'un conseiller, de l'extérieur d'une personne et ensuite dans une autre où il lui suggère de dissimuler ses imperfections physiques: "car vrayement l'exterieur d'une personne la donne fort à cognoistre, oblige fort à l'aymer \& estimer." 38

Ces déplacements, tout en respectant l'essentiel du message sénéquien, créent cependant un espace nouveau de leur valeur. Presque tous les thèmes analysés pour la thèse se sont prêtés à ces variations. Ainsi les propos de Sénèque sur la guerre, contenus dans son traité De la colère, fournissent à Lipse un répertoire d'images pour son éloge de la paix, tandis qu'ils alimentent des considérations plus techniques chez Grotius. Les commentaires de Sénèque sur la tyrannie servent aux absolutistes à souligner les dangers d'un régime instable, aux monarchomaques à rappeler l'illégitimité morale créée par la cruauté, aux scolastiques tels Marquez et Ribadeneyra, ${ }^{39}$ à montrer que le changement de souverain ne signifie pas un changement de régime. Monarchomaques et absolutistes développent, sur les limites du pouvoir, des théories différentes basées sur des prémisses identiques. De telles divergences apparaissent à propos de la loi, de la fortune et de la providence, du rôle du sage dans les affaires de l'Etat, etc.

Une dernière forme de métonymie peut être distinguée. Parfois l'auteur utilise la citation comme le doublet métaphorique de son propos, mais un glissement de sens s'opère imperceptiblement. La menace de la dérivation pèse constamment. Ainsi, lorsque Grotius présente en apparence une condensation métaphorique de ses conceptions sur la propriété des mers en écrivant: "Il est dit que l'Océan est le lien de l'univers et le Boulevard de la Terre (Sénèqe)," 40 il fait plutôt une métonymie de la cause, appuyant un principe économique de la liberté des mers sur le rôle de communication humaine que lui prête Sénèque. C'est une autre métomymie de la cause qu'effectue Fitzherbert lorsqu'il parle de Dieu comme source des épreuves du sage à propos d'un passage où Sénèque faisait plutôt allusion à la Fortune. ${ }^{41}$ D'un avertissement de Jupiter sur certains périls de la crédibilité divine, Marnix met en garde les princes de trop se faire voir. ${ }^{42}$ Une maxime du traité De la colère: “C'est un plaisir d'avoir encore à espérer," sert à Marquez pour inciter le prince à maintenir ses sujets, non dans un sentiment d'espérance, mais dans un état d'attente. ${ }^{43}$ De cette façon, l'auteur mine le sens propre de la citation afin qu'elle réfléchisse mieux son idée. Il instaure une convenance factice soit en négligeant le contexte primitif, soit en élargissant la portée de la réflexion sénéquienne. Il combine la citation avec d'autres éléments auxquels la lie une plus ou moins forte contiguitté de sujet.

Les manières d'articuler le texte ancien s'apparentent donc à la métaphore ou à la métonymie, mais il convient de distinguer des subdivisions au seins de cette dernière figure. Le schéma de l'utilisation des citations serait le suivant: 


\section{6 / Renaissance and Reformation}

CITATION: Métaphore $\longleftrightarrow$ Métonymie

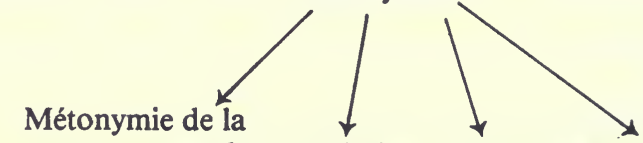

Axe de la $+\quad$ cause ou synecdoque Métalepse Catachrèse Antithèse "fidélité"

Reste à savoir si ces méthodes varient seulement d'un auteur à l'autre, ou au sein même d'une oeuvre. L'utilisation à peu près systématique d'une modalité particulière chez un auteur définirait à la fois son style d'imitation et peut-être la qualité de l'influence qu'un auteur ancien exerce sur lui. Un tel profil est repérable parmi les écrivains de littérature politique que nous avons étudiés. Dans les oeuvres de Robert Bellarmin, ${ }^{44}$ la citation est mise à l'écart du développement et fait l'objet d'un jugement critique qui en dépouille les aspects vieillis ou contraires à la doctrine chrétienne. Cette interrogation du texte ancien constitue sa plus sûre modernisation car elle fait naître la discussion. Même lorsqu'il réfute les thèse des auteurs anciens, il témoigne de sa considération à leur égard. Marnix les respecte beaucoup moins et place ses citations en apposition, sans vraiment les commenter ou les critiquer. Bien souvent, elles sont en disconvenance dissimulée, ou en convenance forcée, avec la démonstration. Marnix sollicite moins l'idée que l'anecdote ou la sentence et il fait flèche de tout bois. La peinture, élogieuse chez Sénèque, de l'austérité des Germains servira à rappeler leur pauvreté avant l'arrivée du christianisme; les habitudes des Egyptiens de regarder le Nil et non le ciel, mentionnées par le même auteur latin, deviendron les exemples de la passivité naturelle du peuple; la mission confiée à Hercule par Jupiter de retrouver les origines de Claude dans l'A pocoloquintose illustrera chez Marnix la théorie qu'il faut accorder le message au messager, l'ouvrage à l'ouvrier. ${ }^{45} \mathrm{C}$ 'est la fonction de la catachrèse qui triomphe ainsi, lorsque d'une idée ancienne est tirée une qualité nouvelle.

Dallington s'en tiendrait plutôt à un usage proche de la métalepse, car chaque aphorisme est suivi d'une liste de citations formant son soutien. Comme elles sont glanées à posteriori et toujours retirées de leur contexte, elles sont tantôt en abruption avec leur sens original, tantôt en disjonction avec l'idée principale de l'aphorisme. Insistant par exemple sur l'importance du temps et des circonstances pour la réussite des affaires, Dallington tirera de la Médée de Sénèque la maxime: "tempori aptari decet," bien éloignée des recommandations stoïciennes sur la constance du sage. Et il ajoute aussitôt un passage des Bienfaits sur les dangers de guerres inutiles causées par la prétention des grands. ${ }^{46}$ Cette seconde citation est mieux accordée que la première à la philosophie de Sénèque, mais moins bien à son propos initial.

Si Lipse nourrit également ses développements d'une pléthore de citations 
d'origine diverse, il respecte davantage leur vérité. La métaphore domine ici, car une forme embellie se superpose à un fond similaire. L'élément comparatif est d'ailleurs explicite par l'emploi des prépositions habituelles: comme, ainsi . . . Quant à Grotius, s'il ouvre dans son texte une parenthèse sur un passage d'un auteur ancien, c'est pour rechercher comme ses prédécesseurs la justesse et l'agrément. Comme eux, il estime et utilise avec bonheur les formules brillantes et concices:

\begin{abstract}
Quant aux choses qui sont acquises dans une guerre injuste, nous avons dit qu'elles doivent être restituées, (. . . ) de quelque manière que ce soit; car personne ne peut transférer à autrui plus de droit qu'il n'en a eu lui-même, disent les auteurs du droit romain, ce que Sénèque explique brièvement: "Personne ne peut donner ce qu'il n'a pas." (Liv. V, De Benef, cap. XII) ${ }^{47}$
\end{abstract}

La répétition du principe est chez Grotius rarement pléonastique. Déplorant ailleurs le sort des mercenaires, il cite cette sentence des Questions naturelles: "On recherche au péril de sa vie à sa procurer des choses qui contribuent à abréger la vie." 48 Grotius n'associe d'ailleurs pas que des idées, il rapproche également des thèmes, reprenant des concepts généraux antiques sur la propriété, la loi naturelle, les peines, la guerre, etc. . . . Grotius ne paraphrase cependant jamais et les principes empruntés servent d'humus nourricier de son propre terreau. Il faut donc lui reconnaître le crédit d'une façon personnelle et novatrice d'appropriation. Les idées antiques ne sont pas confinées à servir d'écho, plus ou moins déformé, à ses propres thèses et servent plutôt de tremplin ou d'assises. Les citations sont davantage la source de métalepse que des métalepses elles-mêmes. Parlant par exemple des gages déposés lors des contrats, Grotius écrit:

Sénèque a eu cela en vue, lorsqu'il a dit que "les uns doivent leur parole, les autres leur tutelle." (De Benef, VII, 19). C'est aussi sur cette règle qu'il aut juger des autres contrats. ${ }^{49}$

Grotius déduit ou induit les implications modernes des théories anciennes. Cette méthode qui engendre moins la discussion comme chez Bellamin que la réflexion, s'apparente à la métalepse, car une idée en provoque une autre. De Nicolas Faret, enfin, nous savons qu'il illustra le mieux la copie, résurrection et intégration brute du texte ancien, ressassement ou réitération, sans discours préliminaires, renvois ou références.

Ainsi malgré l'étonnante diversité des sujets traités, des points de vue et des situations des auteurs, un emprunt reste soumis à des lois d'articulation apparemment identiques à celles du langage; par substitution ou condensation d'une idée par une autre dans le style métaphorique, par combinaison dans l'oeuvre métonymique. Ce sont les deux voies royales, par redoublement ou glissement du sens, d'accès d'une citation au texte contemporain. 
Le rôle de ces deux opérations, sur lesquelles se greffent divers procédés particuliers, est assez fondamental pour que nous en retrouvions la trace dans toutes les influences qui s'exercèrent à la Renaissance. L'ouvrage de Scipione Ammirato ${ }^{50}$ présente cesformes dans l'arrangement des éléments tirés de Tacite. Comme pour tous les auteurs qui puisent abondamment dans le répertoire d'un autre, les procédés varient du synonyme à l'antonyme, bien qu'une forme particulière de relation se dégage plus nettement. Chez Ammirato, la juxtaposition de textes assez identiques est le tour le plus fréquemment manié. Il ne dédaigne cependant pas multiplier les nuances d'un précepte. A propos de la valeur d'entraînement de l'exemple donné par le prince, il accumule des anecdotes de Tacite, Plutarque, TiteLive, pour démontrer que les sujets copient tantôt la vertu de modération du souverain, tantôt son penchant à la gourmandise, tantôt son amour des lettres ou ses qualités d'esprit, et même ses maladies. ${ }^{51}$

L'interaction de deux éléments qui ne sont pas de même nature rappelle le procédé fréquent chez Dallington d'accoler à une maxime un exemple qui ne la concerne qu'au second degré. C'est un autre état de contiguitté que manifeste ce passage:

Le meilleur soldat est celuy qui s'y est le plus exercé, si bien qu'il est nécessaire de s'y accoustumer dès l'enfance. Cecy sera rendu plus intelligible par un passage de Sénèque, auxquel je n'ay pas encore touché, mais qui est fort bien compris dâs les paroles de Tacite qui dit pour troisième raison que Drusus fut envoyé à l'armée en Illyrie pour ne fletrir sa jeunesse dans les desbauches de Rome, outre que par la suitte de l'Histoire on void bien que Drusus n'estoit encore qu'un enfant. ${ }^{52}$

A l'occasion, Ammirato ajoute un jugement sur une anecdote notée sans commentaires chez Tacite. Il blâmera par exemple les libéralités excessives de Néron. Enfin, à l'instar de Grotius, il peut amplifier un sujet et en sonder les ramifications. Défendant dans son traité la sentence: "qu'un bon conseil est un grand gaing," il expose les cas possibles de mauvais effets d'un bon conseil, et vice versa. Il affirme alors que les meilleurs conseils sont ceux qui se tirent de diverses opinions et ajoute:

Car si l'on ne propose qu'un seul advis, il s'y faut tenir: si plusieurs, on choisit le meilleur, \& le plus utile, ce qu'on recognoist aisément en les opposant tous l'un à l'autre. Mais surtout Nullus contationi locus in eo consilio quod non potest laudari nisi per actum. Il ne faut pas encore differer celuy Vbi perniciosor est quies quam temeritas. ${ }^{53}$

Le développement est donc couronné de deux maximes qui forment rétroaction, c'est-à-dire qui reviennent sur l'énoncé pour le confirmer tout en l'élargissant, de sorte que même la citation en fonction de métalepse joue un rôle accessoire de métaphore.

C'est le jeu de ces mécanismes qui assurent aux texte son extension et aux 
idées leur progression. L'éventail des possibilités est cependant restreint. Dans un cas, l'auteur de la Renaissance sélectionne, dans le trésor antique, les thèmes qui concordent le mieux à son propos et les introduit dans son oeuvre, soit par substitution, c'est-à-dire en effaçant sa propre expression comme dans la copie, soit par redoublement, c'est-à-dire en ajoutant la citation à sa pensée. Dans ce remplacement ou ce replacement, l'identité apparente des concepts n'empêche pas un certain nuancement, dû à la qualité d'expressivité et à la fonction d'explicitation de la citation.

Cette réaction, substitutive, devant le texte ancien fait place à une autre, prédicative cette fois, lorsque la similarité des notions est troublée par l'intrusion d'une dimension conceptuelle nouvelle. L'élément introduit dans le procès en cours reste cependant en position de contiguïté, c'est-à-dire qu'il garde des liens plus ou moins lâches avec l'idée principale. Dans ce processus, un sujet en amène un autre grâce à diverses fórmules de dérivation.

Ces deux types de connexion, par similarité, par contiguïté, révèlent deux styles d'imitation, le premier lié à la périphrase puisque l'auteur reprend une idée de manière un peu détournée et surtout plus fastueuse, le second allié à l'épiphrase, lorsqu'on fait entendre une chose par une autre. La préférence de la majorité des auteurs dont Marnix et Faret va à la relation interne de commutation simplement formelle. La citation sert à valoriser l'élocution. Seuls Bellarmin, Lipse et Grotius favoriseront plutôt la relation externe d'assemblage, qui sert le mieux la réflexion.

\section{Université d'Ottawa}

\section{Notes}

1 S. Morawski, "This Basic Functions of Quotation," dans Signe, Langage, Culture (La Haye, 1970), p. 690-705.

2 Per Aage Brandt, "La Pensée du texte (de la littéralité de la littérarité)," dans Essais de la théorie du texte (Paris, Galilée, 1973), p. 208 et ss. Brandt s'intéresse à la symbolisation et à la transgression créées par les citations.

D. Maingueneau, Initiation aux méthodes de l'analyse du discours (Paris, Hachette, 1976), p. 125 et ss. Maingueneau distingue pour sa part quatre fonctions des citations: relique, preuve, épigraphe, culture.

3 B. Beugnot, "Un aspect textuel de la réception critique: le citation," dans Oeuvres et critiques, I, 2 (1976), p. 5-19.

4 A. Compagnon, La Seconde Main ou le travail de la citation (Paris, Seuil, 1979), 414 pages.

5 "Le moyen âge, lui, avait établi autour du livre quatre fonctions distinctes: le scriptor (qui recopiait sans rien ajouter), le compilator (qui n'ajoutait jamais du sien), le commentator (qui n'intervenait de lui-même dans le texte recopié que pour le rendre intelligible) et enfin l'auctor (qui donnait ses propres idées, en s'appuyant toujours sur d'autres autorités). Un tel système, établi explicitement à la seule fin d'être "fidèle" au texte ancien, seul Livre reconnu (peut-on imaginer plus grand "respect" que celui du moyen âge pur Aristote ou Priscine?), un tel système 
a cependant produit une "interprétation" de l'Antiquité que la modernité s'est empressée de récuser et qui apparaîtrait à notre critique "objective" parfaitement "délirante." R. Barthes, Critique et vérité (Paris, Seuil, 1966), p. 76-77. Pour une étude des termes auctor, actor et magister dans les textes du XII ${ }^{\mathrm{e}}$ siècle, cf. Marie-Dominique Chenu, "Auctor, actor, autor," dans Bulletin du Cange, Archivium Latinitatis Medii Aevi, 3 (1927), 81-86 et son étude dans son ouvrage La Théologie au $12^{\ell}$ siècle (Paris, 1976), p. 351-365. Et pour une analyse de la tradition manuscrite, l'article de David Hult, "Gui de Mori, lecteur médiéval," paru dans Incidences, vol. IV, $\mathrm{n}^{\circ} 3$.

6 Montaigne, Essais (Paris, Garnier, 1962), L. III, chapitre 5.

7 Citées par C.-J. Friedrich, dans la préface de son édition de l'ouvrage de Johannes Althusius, Politica Methodice Digesta (Cambridge, Harvard University Press, 1932), 427 pages. (1 ${ }^{\text {re }}$ édition du Politica: Groningue, 1603).

8 Montaigne, op. cit., L. II, chapitre 12.

9 A.-P. Filière, Discours contre les citations du grec et du latin ès plaidoyers de ce temps (Paris, 1610). Cité par B. Beugnot.

10 Juste Lipse, Les Politiques (Genève, Pierre et Jaques Chovet, 1613), 634 pages. Pas de numérotation pour la Préface et pour l'épître $A$ u lecteur. (Première édition des Politiques: Lyon, 1589.)

11 Ibid.

12 Jean Bodin, Les Six Livres de la République (Lyon, J. du Puys, 1579), p. 676. (1 ${ }^{\text {re }}$ édition: 1576.)

13 Jean de Marnix, Résolutions politiques et maximes d'Estat (Brusselles, François Vivien, 1632). ( $1^{\text {re }}$ édition: Bruxelles, 1612.)

14 Juste Lipse, op. cit., Vers liminaires de l'ouvrage.

15 Hugo de Groot, dit Grotius, Le Droit de la guerre et de la paix, Nouvelle trad. précédée d'un essai sur Grotius par P. Pradier-Fodéré (Paris, Guillaumin, 1867), 3 volumes, in- $8^{\circ}$. ( $1^{\text {re }}$ édition: Paris, 1625.)

16 Selon l'expression “foreign body," utilisée par A. Wierzbicka, dans son article, “Descriptions or quotations," dans Signe, Langage, Culture, p. 627.

17 Sénèque, Lettres à Lucilius (Paris, Les Belles Lettres, 1962-1965), XVII, 108, 29.

18 Roman Jakobson, “Two Aspects of Language and Two Types of Aphasic Disturbances," dans Selected Writings, vol. 2 (Mouton, The Hague, 1971), p. 239-259. L'article a été traduit en français par A. Adler et N. Ruwet et a paru sous le titre "Deux aspects du langage et deux types d'aphasie," dans Les Temps modernes, janvier-juin 1962, $17^{\mathrm{e}}$ année, $\mathrm{n}^{\circ} 188$, p. 853-880.

19 J. de Marnix, op. cit., p. 262.

20 Juste Lipse, épître “Au lectuer" des Politiques. L'épître ne comporte pas de numérotation. Les vers cités sont tirés des Troyennes de Sénèque.

21 P. Juan de Marquez, L'Homme d'Estat chrestien, tiré des vies de Moyse et Josué Princes du peuple de Dieu. Trad. D. Virion, Nancy, (Jacob Granich, 1621). (1 ${ }^{\text {re }}$ édition: Pampelune, 1615.)

22 Juan de Marnix, op. cit., p. 98.

23 Ibid., p. 629.

24 Nicolas Faret, Des vertus nécessaires à un prince pour bien gouverner ses sujets (Paris, Toussaint du Bray, 1623). Dans une situation semblable, Montaigne préféra indiquer sa source. Il envoya à sa femme la lettre de consolation que Plutarque fit parvenir à la sienne en écrivant: "je desroberay icy les mots mesmes de Plutarque qui valent mieux que les miens.” Lettre citée par Claude Blouin, dans Influence des Vies de Plutarque sur le premier livre des Essais de Montaigne (thèse de maîtrise, Université de Montréal, 1968), p. 172.

25 Sir Robert Dallington, Aphorismes Civill and Militarie (London, Edward Blount, 1613), p. 92

26 Juan de Marnix, op. cit., p. 412.

27 Selon l'expression d'André Stegmann, dans Le Tacitisme, “Il Pensiero Politico," 1970.

28 Juan de Marnix, op. cit., p. 312 et 370; Sir R. Dallington, op. cit., p. 15.

29 Thomas Fitzherbert, The first and second part of a Treatise concerning Policy and Religion (Douay, J. Heigham, 1615), un volume. ( $1^{\text {re }}$ édition de la $1^{\text {re }}$ partie: Douai, 1606; de la $2^{\mathrm{e}}$ partie: Douai, 1610.) 
30 Philippe de Duplessis Mornay, De la puissance légitime du Prince sur le peuple et du peuple sur le Prince. Escrit en latin par Estienne Junius Brutus. Trad. (François Estienne), s. 1., 1581 . (1 $1^{\mathrm{re}}$ édition: Bâle, 1579.) Note: Cet ouvrage est parfois attribué à Hubert Languet ou à Théodore de Bèze.

François Hotman, La France-Gaule ou Gaule françoise, texte paru dans les Mémoires de l'Estat de France sous Charles neuviesme, tome I (Meidelbourg, Henrich Wolf, 1577), p. 375 et ss.

31 Etienne de la Boétie, De la Servitude volontaire, ou le Contr'un. Avec les notes de M. Coste. Paris, P. Daubrée et Cailleux, 1835. (1 ${ }^{\mathrm{re}}$ édition dans les Mémoires de l'Estat de France, tome II, Meidelbourg, 1578).

32 Charles Dumarsais, Des tropes, Slatkine Reprints, Genève, cité par Michel Charles, "Le Discours des figures," dans Poétique, 1973, n 15, p. 362.

33 Cité par M. Charles, op. cit., p. 362-363.

34 La métalepse est ainsi définie dans Gradus: "Faire entendre une chose par une autre, qui la précède, la suit ou l'accompagne, en est . . . une circonstance quelconque, ou enfin s'y rattache ou s'y rapporte de manière à la rappeler aussitôt à l'esprit. Fontanier, p. 127-128." Bernard Dupriez, Gradus. Les procédés littéraires. (Dictionnaire) (Paris, Union générale d'Editions, “10/18," 1980), p. 284.

35 Grotius, op. cit., L, III, p. 167.

36 "HYPALLAGE, en grec Y $\pi \alpha \lambda \lambda \alpha \eta \eta$, transposition, renversement; de $\xi \pi 0$, sous ou de, et de $\alpha \lambda \lambda \alpha \gamma \eta$, changement, dérivé de $\alpha \lambda \lambda \alpha \tau \tau \omega$, changer. On entend par ce mot le transport fait à quelqu'un des objets d'une phrase, de ce qui ne semble réellement convenir qu'à un autre objet avec lequel il s'y trouve en rapport."

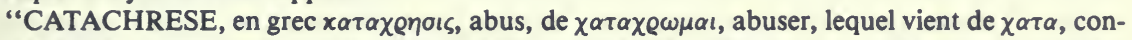
tre, et de $\chi \varrho \omega \mu \alpha$, user: abuser, c'est en effet, contre-user, c'est faire un usage tout contraire à celui qu'on devrait faire." Fontanier, Les Figures du discours, p. 266 et 213. (Paris, Flammarion, 1968, 505 pages.)

Note: L'emploi de ces termes de rhétorique répond à ces impératifs d'interprétation et de classification. Aussi ces termes ne recouvrent-ils pas très exactement leurs définitions dans les traités de rhétorique.

37 Fitzherbert, op. cit., p. 89 et 392.

38 J. de Marnix, op. cit., p. 112.

39 Pedro de Ribadeneyra, Traité de la Religion que doit suivre le prince chrestien et des vertus qu'il doit avoir pour bien conserver son Estat. Contre la doctrine de Nicolas Machiavel et des Politiques de nostre temps. Trad. Antoine de Balinghem (Douay, Jean Bogart, 1610). (1 ${ }^{\text {re }}$ édition: Madrid, 1595.)

40 Grotius, op. cit., L. III, p. 396.

41 T. Fitzherbert, op. cit., p. 212.

42 J. de Marnix, op. cit., p. 191.

43 J. de Marquez, op. cit., p. 90.

44 Robert Bellarmin, Le Monarque parfait ou le Devoir d'un prince chrétien. Trad. Jean de Lannel (Paris, Sebastien Cramoisy, 1625). (1 ${ }^{\text {re }}$ édition: Rome, 1619.)

45 J. de Marnix, op. cit., p. 14, 51 et 98.

46 Sir R. Dallington, op. cit., p. 127.

47 Grotius, op. cit., L, III, p. 329.

48 Ibid., L. III, p. 598.

49 Ibid., L. II, p. 166.

50 Scipione Ammirato, Politiques sur les oeuvres de C. Tacitus. Trad. I. Baudoin (Paris, Jean

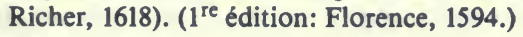

51 Ibid., p. 46-47.

52 Ibid., p. 36.

53 Ibid., p. 87. 